\title{
Imaging the water snowline in a protostellar envelope with $\mathrm{H}^{13} \mathrm{CO}^{+\star, \star \star}$
}

\author{
Merel L. R. van 't Hoff' ${ }^{1}$, Magnus V. Persson², Daniel Harsono' ${ }^{1}$, Vianney Taquet ${ }^{1,3}$, Jes K. Jørgensen ${ }^{4}$, \\ Ruud Visser ${ }^{5}$, Edwin A. Bergin ${ }^{6}$, and Ewine F. van Dishoeck ${ }^{1,7}$ \\ ${ }^{1}$ Leiden Observatory, Leiden University, PO Box 9513, 2300 RA Leiden, The Netherlands \\ e-mail: vthoff@strw.leidenuniv.nl \\ 2 Department of Space, Earth and Environment, Chalmers University of Technology, Onsala Space Observatory, 43992 Onsala, \\ Sweden \\ ${ }^{3}$ INAF, Osservatorio Astrofisico di Arcetri, Largo E. Fermi 5, 50125 Firenze, Italy \\ ${ }^{4}$ Centre for Star and Planet Formation, Niels Bohr Institute \& Natural History Museum of Denmark, University of Copenhagen, \\ Øster Voldgade 5-7, 1350 Copenhagen K, Denmark \\ ${ }^{5}$ European Southern Observatory, Karl-Schwarzschild-Str. 2, 85748 Garching, Germany \\ ${ }^{6}$ Department of Astronomy, University of Michigan, 1085 S. University Ave., Ann Arbor, MI 48109-1107, USA \\ ${ }^{7}$ Max-Planck-Institut für Extraterrestrische Physik, Giessenbachstrasse 1, 85748 Garching, Germany
}

Received 27 July 2017 / Accepted 3 January 2018

\begin{abstract}
Context. Snowlines are key ingredients for planet formation. Providing observational constraints on the locations of the major snowlines is therefore crucial for fully connecting planet compositions to their formation mechanism. Unfortunately, the most important snowline, that of water, is very difficult to observe directly in protoplanetary disks because of the close proximity of this snowline to the central star.

Aims. Based on chemical considerations, $\mathrm{HCO}^{+}$is predicted to be a good chemical tracer of the water snowline because it is particularly abundant in dense clouds when water is frozen out. This work aims to map the optically thin isotopolog $\mathrm{H}^{13} \mathrm{CO}^{+}$toward the envelope of the low-mass protostar NGC 1333-IRAS2A, where the snowline is at a greater distance from the star than in disks. Comparison with previous observations of $\mathrm{H}_{2}^{18} \mathrm{O}$ show whether $\mathrm{H}^{13} \mathrm{CO}^{+}$is indeed a good tracer of the water snowline.

Methods. NGC 1333-IRAS2A was observed using the NOrthern Extended Millimeter Array (NOEMA) at $\sim 0^{\prime} 9$ resolution, targeting the $\mathrm{H}^{13} \mathrm{CO}^{+} J=3-2$ transition at $260.255 \mathrm{GHz}$. The integrated emission profile was analyzed using 1D radiative transfer modeling of a spherical envelope with a parametrized abundance profile for $\mathrm{H}^{13} \mathrm{CO}^{+}$. This profile was validated with a full chemical model.

Results. The $\mathrm{H}^{13} \mathrm{CO}^{+}$emission peaks $\sim 2^{\prime \prime}$ northeast of the continuum peak, whereas $\mathrm{H}_{2}^{18} \mathrm{O}$ shows compact emission on source. Quantitative modeling shows that a decrease in $\mathrm{H}^{13} \mathrm{CO}^{+}$abundance by at least a factor of six is needed in the inner $\sim 360$ AU to reproduce the observed emission profile. Chemical modeling indeed predicts a steep increase in $\mathrm{HCO}^{+}$just outside the water snowline; the $50 \%$ decrease in gaseous $\mathrm{H}_{2} \mathrm{O}$ at the snowline is not enough to allow $\mathrm{HCO}^{+}$to be abundant. This places the water snowline at $225 \mathrm{AU}$, further away from the star than expected based on the $1 \mathrm{D}$ envelope temperature structure for NGC 1333-IRAS2A. In contrast, DCO ${ }^{+}$ observations show that the $\mathrm{CO}$ snowline is at the expected location, making an outburst scenario unlikely.

Conclusions. The spatial anticorrelation of $\mathrm{H}^{13} \mathrm{CO}^{+}$and $\mathrm{H}_{2}^{18} \mathrm{O}$ emission provide proof of concept that $\mathrm{H}^{13} \mathrm{CO}^{+}$can be used as a tracer of the water snowline.
\end{abstract}

Key words. ISM: individual objects: NGC 1333-IRAS2A - ISM: molecules - astrochemistry - stars: protostars submillimeter: planetary systems

\section{Introduction}

Water is probably the molecule that appeals most to our imagination, as it is essential for life as we know it. In star-forming regions, water plays an important role as a coolant of warm gas, a dominant carrier of oxygen, and a major constituent of icy grain mantles deep inside a planet-forming disk (see, e.g., Melnick 2009; Bergin \& van Dishoeck 2012; van Dishoeck et al. 2013, for reviews). The transition from water being frozen

\footnotetext{
* The NOEMA data are only available at the CDS via anonymous ftp to cdsarc.u-strasbg.fr $(130.79 .128 .5)$ or via http://cdsarc.u-strasbg.fr/viz-bin/qcat?]/A+A/613/A29

$\star \star$ Based on observations carried out with the IRAM NOEMA interferometer. IRAM is supported by INSU/CNRS (France), MPG (Germany), and IGN (Spain).
}

out onto dust grains to being predominantly present in the gas phase occurs at the water snowline: the midplane radius at which $50 \%$ of the water is in the gas phase and $50 \%$ is in ice. Since the selective freeze-out of the major oxygen carrier alters the elemental $\mathrm{C} / \mathrm{O}$ ratio in both gas and ice, the bulk chemical composition of planets depends on their formation location with respect to the freeze-out radius of water (e.g., Öberg et al. 2011; Madhusudhan et al. 2014; Ali-Dib et al. 2014; Ali-Dib 2017; Walsh et al. 2015; Mordasini et al. 2016; Eistrup et al. 2016; Booth et al. 2017). In addition, planetesimal formation is thought to be significantly enhanced in this region (e.g., Stevenson \& Lunine 1988; Schoonenberg \& Ormel 2017). The exact location and time evolution of the water snowline in protostellar systems is thus a crucial ingredient in planet formation. 
Unfortunately, the water snowline is very hard to observe directly in protoplanetary disks. Because of the large binding energy of $\mathrm{H}_{2} \mathrm{O}$, the transition from ice to gas happens a few AU from the young star where the midplane temperature exceeds $\sim 100-200 \mathrm{~K}$ (depending on the vapor pressure). For the nearest star-forming regions, this would already require angular resolutions of $\lesssim 00^{\prime} 01$. Emission originating from cold water $\left(E_{\text {up }}<100 \mathrm{~K}\right)$ has been detected in the disk around TW Hya with the Herschel Space Observatory (Hogerheijde et al. 2011; Zhang et al. 2013), but the large Herschel beam $\left(10-45^{\prime \prime}\right)$ could not spatially resolve the snowline. Furthermore, the only thermal water lines that can be observed from the ground (except for the $\mathrm{H}_{2} \mathrm{O}$ line at $183 \mathrm{GHz}$ ) are lines from the less abundant isotopolog $\mathrm{H}_{2}^{18} \mathrm{O}$. As such, even ALMA will have great difficulty locating the water snowline in protoplanetary disks.

An alternative approach is to use chemical imaging, as has been carried out for the $\mathrm{CO}$ snowline. Because $\mathrm{CO}$ is highly volatile, its snowline is located tens of $\mathrm{AU}$ from the central star (around 20 K; see, e.g., Burke \& Brown 2010). Although this is far enough to be spatially resolved with ALMA, locating it directly remains difficult; since CO line emission is generally optically thick, it does not reveal the cold disk midplane. Reactions with gaseous $\mathrm{CO}$ are the main destruction route for $\mathrm{N}_{2} \mathrm{H}^{+}$, therefore $\mathrm{N}_{2} \mathrm{H}^{+}$is expected to be abundant only when $\mathrm{CO}$ is frozen out. Taking simple chemical considerations into account, an upper limit for the $\mathrm{CO}$ snowline location can therefore be derived from $\mathrm{N}_{2} \mathrm{H}^{+}$emission (Aikawa et al. 2015; van 't Hoff et al. 2017), as has been performed for the disks around TW Hya and HD 163296 (Qi et al. 2013, 2015), and several protostellar envelopes (Jørgensen 2004; Anderl et al. 2016).

For young protostars, the emission of complex organic molecules (COMs) has been used as tracer of the inner region that is warm enough to sublimate water ice, the so-called hot core, because these species are expected to be trapped in water ice. However, the exact binding energy, and thus spatial extent, differs for different molecules. Compact COM emission has been detected toward NGC 1333-IRAS2A (hereafter IRAS2A) extending between $\sim 0$.' 4 and $\sim 11^{\prime \prime} 0$ (e.g., Jørgensen et al. 2005; Maret et al. 2014; Maury et al. 2014), similar to the size ( 0.'8) of the $\mathrm{H}_{2}^{18} \mathrm{O}$ emission (Persson et al. 2012). The exact relationship between COMs and the water snowline remains unclear, however. For example, the extent of methanol emission in IRAS2A is half the size of the $\mathrm{H}_{2}^{18} \mathrm{O}$ emission (Maret et al. 2014), while it is twice the size in NGC 1333-IRAS4A (Anderl et al. 2016). In addition, Persson et al. (2012) concluded that $\mathrm{C}_{2} \mathrm{H}_{5} \mathrm{CN}$ is likely not related to sublimation of water ice. Since the detection of COMs is very difficult in mature protoplanetary disks, where only methyl cyanide and methanol have been observed so far (Öberg et al. 2015; Walsh et al. 2016), COMs are not suited as tracers of the water snowline in these systems.

The best candidate to chemically trace the water snowline is $\mathrm{HCO}^{+}$because its most abundant destroyer in warm dense gas is gaseous $\mathrm{H}_{2} \mathrm{O}$, that is,

$\mathrm{H}_{2} \mathrm{O}+\mathrm{HCO}^{+} \rightarrow \mathrm{CO}+\mathrm{H}_{3} \mathrm{O}^{+}$.

A strong decline in $\mathrm{HCO}^{+}$is thus expected when $\mathrm{H}_{2} \mathrm{O}$ desorbs off the dust grains (Phillips et al. 1992; Bergin et al. 1998). Atacama Large Millimeter/submillimeter Array (ALMA) observations of the optically thin isotopolog $\mathrm{H}^{13} \mathrm{CO}^{+}$toward the Class 0 protostar IRAS 15398-3359 indeed revealed ring-shaped emission (Jørgensen et al. 2013). The spatial distribution is consistent with destruction by water in the innermost region, but the inner radius of the $\mathrm{H}^{13} \mathrm{CO}^{+}$emission is further out than expected. This can be explained if the temperature has recently been higher, that is, if the source has undergone a luminosity outburst. Follow-up observations by Bjerkeli et al. (2016) did not detect the $\mathrm{H}_{2}^{18} \mathrm{O} 4_{14}-3_{21}$ high excitation transition $\left(E_{u p}=322 \mathrm{~K}\right)$. However, an HDO transition with lower upper level energy $\left(E_{u p}=22 \mathrm{~K}\right)$ was clearly detected. Although HDO emission is also present in the outflow lobes, the observations are consistent with the $\mathrm{H}_{2} \mathrm{O}-\mathrm{HCO}^{+}$anticorrelation scenario.

Protostellar envelopes are good targets to test the $\mathrm{H}^{13} \mathrm{CO}^{+}-\mathrm{H}_{2}^{18} \mathrm{O}$ anticorrelation. Because of higher luminosity (due to higher accretion rates) and lower vapor pressure, the snowline is located further away from the star than in protoplanetary disks (10 s-100 AU instead of a few AU; Harsono et al. 2015; Cieza et al. 2016). In addition, $\mathrm{H}_{2}^{18} \mathrm{O}$ has already been observed toward four of these objects (Jørgensen \& van Dishoeck 2010; Persson et al. 2012, 2013). The only thing lacking is thus high-spatial resolution images of $\mathrm{H}^{13} \mathrm{CO}^{+}$. Here, we present NOEMA observations of $\mathrm{H}^{13} \mathrm{CO}^{+}$toward one source, IRAS2A, and compare these to the $\mathrm{H}_{2}^{18} \mathrm{O}$ emission presented by Persson et al. (2012). IRAS2A is a deeply embedded Class 0 protostar in the NGC 1333 region of the Perseus molecular cloud. The quadruple outflows suggest that it is a close binary (Jørgensen et al. 2004a), which has recently been confirmed (0'.6 separation; Tobin et al. 2015). Similar to Persson et al. (2012) we adopt a distance of 250 pc (Enoch et al. 2006).

The observations and results are presented in Sects. 2 and 3, and compared with the $\mathrm{H}_{2}^{18} \mathrm{O}$ observations from Persson et al. (2012). In Sect. 4, the integrated $\mathrm{H}^{13} \mathrm{CO}^{+}$emission is analyzed using $1 \mathrm{D}$ radiative transfer modeling of a spherical envelope with a parametrized $\mathrm{H}^{13} \mathrm{CO}^{+}$abundance profile. In addition, this abundance profile is validated against the outcome of a full chemical network and low-resolution $\mathrm{DCO}^{+}$observations. The main conclusions, including that we confirm the predicted anticorrelation between $\mathrm{H}^{13} \mathrm{CO}^{+}$and $\mathrm{H}_{2}^{18} \mathrm{O}$, are summarized in Sect. 5.

\section{Observations}

IRAS2A $\left(\alpha(2000)=03^{\mathrm{h}} 28^{\mathrm{m}} 55^{\mathrm{s}} .58 ; \delta(2000)=31^{\circ} 14^{\prime} 37^{\prime \prime} \cdot 10\right)$ was observed using the NOrthern Extended Millimeter Array (NOEMA) on December 1, 2015 (C configuration), and April 9, 2016 (B configuration) for a total of $2.3 \mathrm{~h}$ on source in B configuration and $1.2 \mathrm{~h}$ in $\mathrm{C}$ configuration. Combining the observations in the different configurations, the data cover baselines from 16.8 to $456.9 \mathrm{~m}(14.5$ to $396.6 \mathrm{k} \lambda)$. The receivers were tuned to the $\mathrm{H}^{13} \mathrm{CO}^{+} J=3-2$ transition at $260.255 \mathrm{GHz}(1.15 \mathrm{~mm})$, and the narrowband correlator was set up with one unit (bandwidth of $40 \mathrm{MHz}$ ) centered on this frequency providing a spectral resolution of $0.078 \mathrm{MHz}\left(0.09 \mathrm{~km} \mathrm{~s}^{-1}\right)$. In addition, the WideX correlator was used, covering a $3.6 \mathrm{GHz}$ window $(259.2-262.8 \mathrm{GHz})$ at a resolution of $1.95 \mathrm{MHz}\left(2.2-2.3 \mathrm{~km} \mathrm{~s}^{-1}\right)$.

Calibration and imaging were performed using the CLIC and MAPPING packages of the IRAM GILDAS software ${ }^{1}$. The standard calibration procedure was followed using the quasars $3 C 454.3$ and 3 C 84 to calibrate the bandpass, $0333+321$ to calibrate the complex gains, and the point sources MWC349 and $\mathrm{LkH} \alpha 101$ to calibrate the absolute flux scale. The continuum visibilities were constructed from line-free channels in the WideX spectrum, and the continuum was subtracted from the

http://www . iram. fr/IRAMFR/GILDAS 


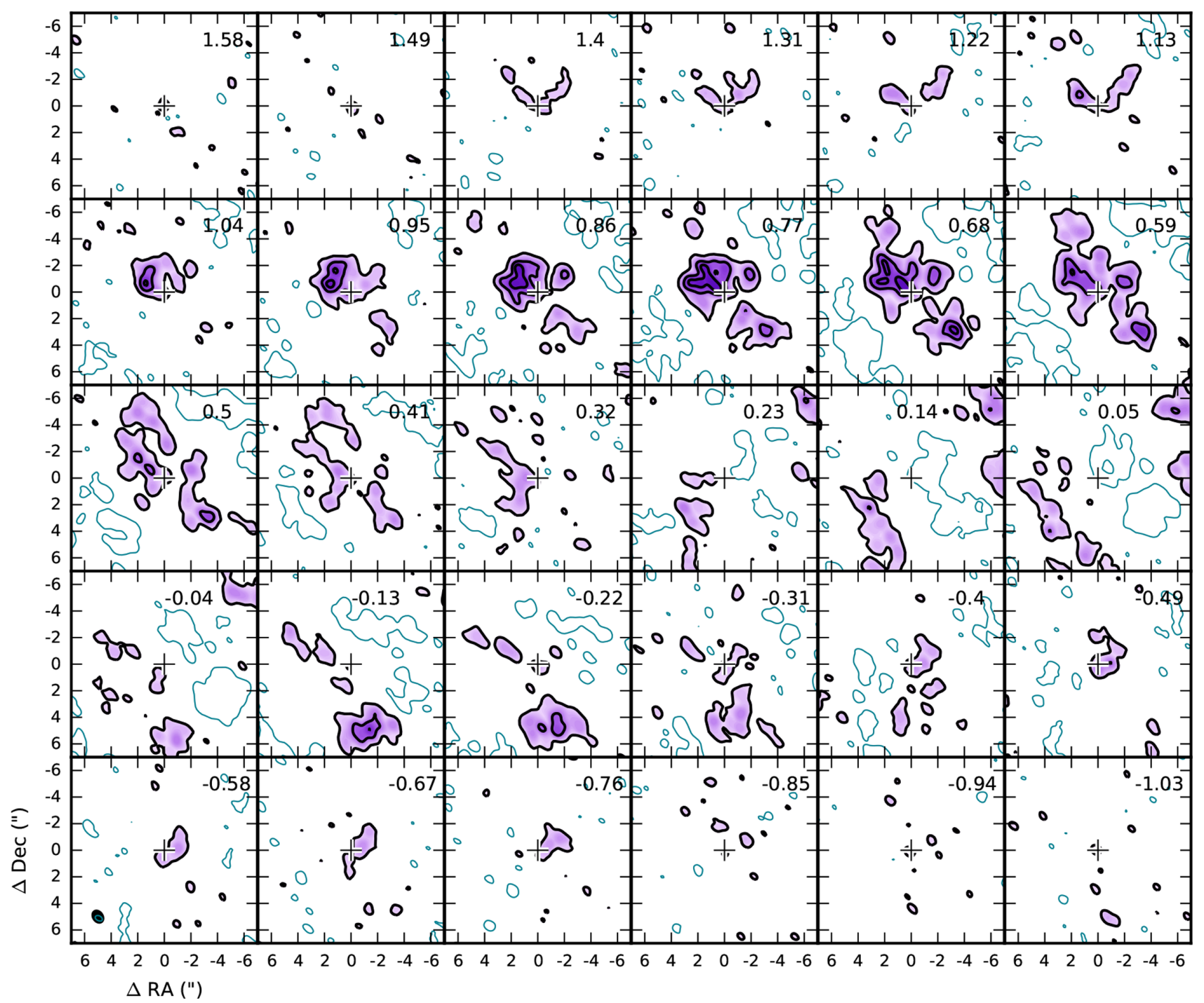

Fig. 1. Channel maps for the $\mathrm{H}^{13} \mathrm{CO}^{+} J=3-2$ transition. Black contours are in steps of $10 \sigma$, starting from a $3 \sigma$ level $\left(30 \mathrm{mJy}\right.$ beam ${ }^{-1}$ in $0.09 \mathrm{~km} \mathrm{~s}^{-1}$ channels), and the $-3 \sigma$ contours are shown in blue. The peak intensity is $324 \mathrm{mJy} \mathrm{beam}^{-1}$ at $7.68 \mathrm{~km} \mathrm{~s}^{-1}\left(v_{\mathrm{lsr}}+0.68 \mathrm{~km} \mathrm{~s}^{-1}\right)$. The continuum peak position is indicated with a cross and the beam is shown in the bottom left corner of the bottom left panel. Channel velocities with respect to the systemic velocity of $v_{\mathrm{lsr}} \approx 7.0 \mathrm{~km} \mathrm{~s}^{-1}$ are listed in the top right corner of each panel. For the adopted distance to IRAS2A, $1^{\prime \prime}$ corresponds to $250 \mathrm{AU}$.

Table 1. Overview of the molecular line observations toward IRAS2A.

\begin{tabular}{lcccc}
\hline \hline Transition & $\begin{array}{c}\text { Frequency } \\
(\mathrm{GHz})\end{array}$ & $\begin{array}{c}E_{\text {up }} / k \\
(\mathrm{~K})\end{array}$ & $\begin{array}{c}\text { Beam } \\
\left({ }^{\prime \prime}\right)\end{array}$ & $\begin{array}{c}\Delta v^{a} \\
\left(\mathrm{~km} \mathrm{~s}^{-1}\right)\end{array}$ \\
\hline $\mathrm{H}^{13} \mathrm{CO}^{+} J=3-2$ & 260.255 & 25 & $0.93 \times 0.68$ & 0.09 \\
$\mathrm{H}_{2}^{18} \mathrm{O}_{1,3-2,0}$ & 203.408 & 204 & $0.87 \times 0.72$ & 0.115 \\
$\mathrm{DCO}^{+} J=2-1$ & 144.068 & 10 & $2.1 \times 1.7$ & 4.06 \\
\hline
\end{tabular}

Notes. ${ }^{(a)}$ Velocity resolution.

line data before imaging. Both the line and continuum data were imaged using default robust weighting. For $\mathrm{H}^{13} \mathrm{CO}^{+}$this resulted in a $0 .^{\prime} 93 \times 00^{\prime} 68\left(\mathrm{PA}=36^{\circ}\right)$ beam, which is comparable to the beam of the $\mathrm{H}_{2}^{18} \mathrm{O}$ observations (see below), and a rms of $10 \mathrm{mJy}^{\text {beam }}{ }^{-1}$ in $0.09 \mathrm{~km} \mathrm{~s}^{-1}$ channels. The continuum image has a $0 .^{\prime} 95 \times 0 . .^{\prime} 65\left(\mathrm{PA}=27^{\circ}\right)$ beam. The continuum flux obtained from a Gaussian fit to the visibilities is $347 \mathrm{mJy}$.
This is consistent with previous observations between 0.8 and $1.47 \mathrm{~mm}$ (Jørgensen et al. 2007; Persson et al. 2012; Taquet et al. 2015) within the $20 \%$ flux calibration error, assuming a power-law spectrum for thermal dust emission $\left(F_{v} \propto v^{\alpha}\right.$, with $\alpha \sim 2.0-2.4)$. The $(u, v)$-fitted continuum peak position $\left(\alpha(2000)=03^{\mathrm{h}} 28^{\mathrm{m}} 55^{\mathrm{s}} .57 ; \delta(2000)=31^{\circ} 14^{\prime} 36^{\prime \prime} \cdot 92\right)$ is located $\sim 0$.' 1 south of the bright component of the 0 . $^{\prime} 6$ binary identified by Tobin et al. (2015).

The $\mathrm{H}_{2}^{18} \mathrm{O} 3_{1,3}-2_{2,0}$ transition at $203.408 \mathrm{GHz}(1.47 \mathrm{~mm})$ was observed using the Plateau de Bure Interferometer (PdBI) in December 2009 and March 2010 and was presented by Persson et al. (2012). The data have a spectral resolution of $0.087 \mathrm{MHz}$ $\left(0.115 \mathrm{~km} \mathrm{~s}^{-1}\right)$ and a resulting beam size of $0.87 \times 0.72$ $\left(\mathrm{PA}=63.5^{\circ}\right)$ using natural weighting.

In addition, IRAS2A was observed using the PdBI in July, August, and November 2010 and March 2011 in the C and D configurations as part of a study of complex organic molecules toward low-mass protostars (Taquet et al. 2015). The WideX backends were used at $\sim 145 \mathrm{GHz}$ providing a 

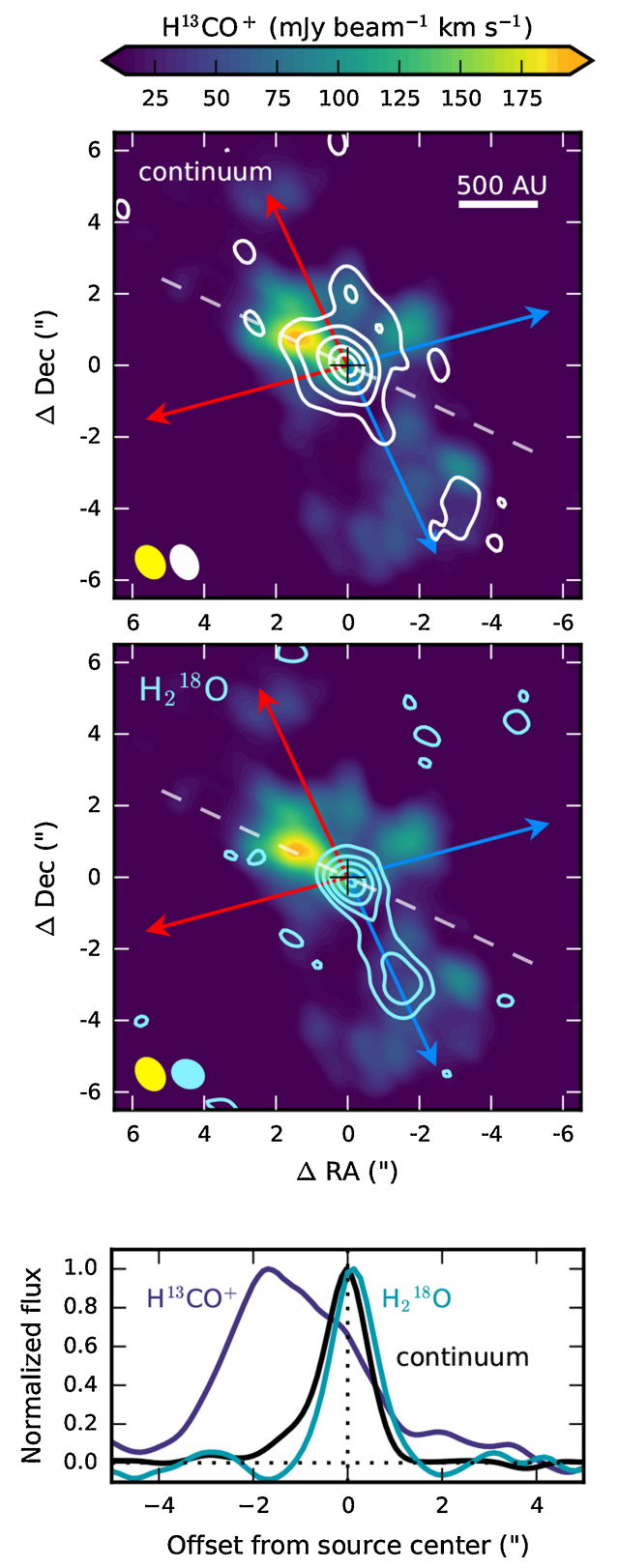

Fig. 2. Integrated intensity map for the $\mathrm{H}^{13} \mathrm{CO}^{+} J=3-2$ transition (color scale) toward IRAS2A. The $1.2 \mathrm{~mm}$ continuum is overlaid in white contours (top panel) and the $\mathrm{H}_{2}^{18} \mathrm{O} 3_{1,3}-2_{2,0}$ transition in blue contours (middle panel). The continuum contours are $1.8(1 \sigma) \times[3,10$, $25,50,75] \mathrm{mJy}_{\text {beam }}^{-1}$, and the $\mathrm{H}_{2}^{18} \mathrm{O}$ contours are $9.8(1 \sigma) \times[3,8,15$, $25,35] \mathrm{mJy}_{\text {beam }}{ }^{-1} \mathrm{~km} \mathrm{~s}^{-1}$. The beams are depicted in the bottom left corner. The position of the continuum peak is indicated by a black cross (the close binary is unresolved in these data) and the outflow axes by red and blue arrows. The integrated intensities along the dashed white line are shown in the bottom panel, normalized to their maximum value. The zero flux level and source position are indicated by dotted lines.

bandwidth of $3.6 \mathrm{GHz}$ with a spectral resolution of $1.95 \mathrm{MHz}$ $\left(\sim 3.5-4 \mathrm{~km} \mathrm{~s}^{-1}\right)$. One of the targeted lines was the $\mathrm{DCO}^{+}$ $J=2-1$ transition at $144.068 \mathrm{GHz}$. Phase and amplitude were calibrated by performing regular observations of the nearby point sources $3 \mathrm{C} 454.3,3 \mathrm{C} 84$, and $0333+321$. Imaging with natural weighting resulted in a $2^{\prime \prime} .1 \times 1^{\prime \prime}$. $\left(\mathrm{PA}=-155^{\circ}\right)$ beam.

An overview of the observed molecular lines and their parameters is provided in Table 1.

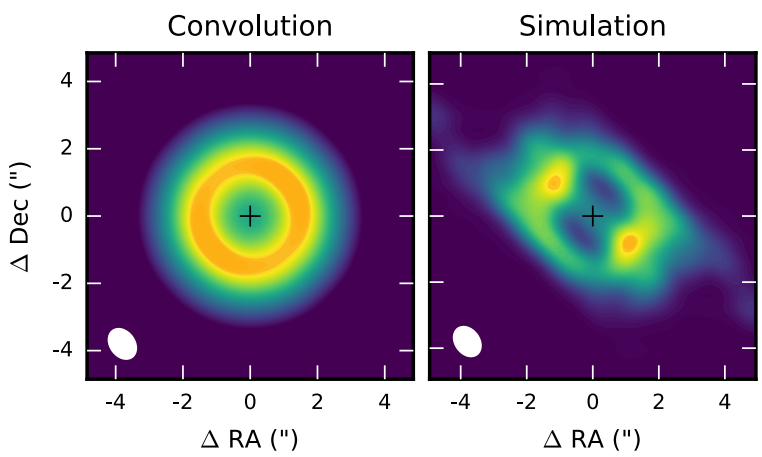

Fig. 3. Integrated intensity maps for a spherically symmetric ringshaped $\mathrm{H}^{13} \mathrm{CO}^{+}$distribution. Emission simulated with the radiative transfer code Ratran is convolved with the observed beam (left panel), and simulated with the $(u, v)$ coverage of the NOEMA observations (right panel). The southern peak in the simulations is probably not observed owing to presence of water in the outflow (see text for details). The continuum peak is denoted with a cross, and the beam is shown in the bottom left corner.

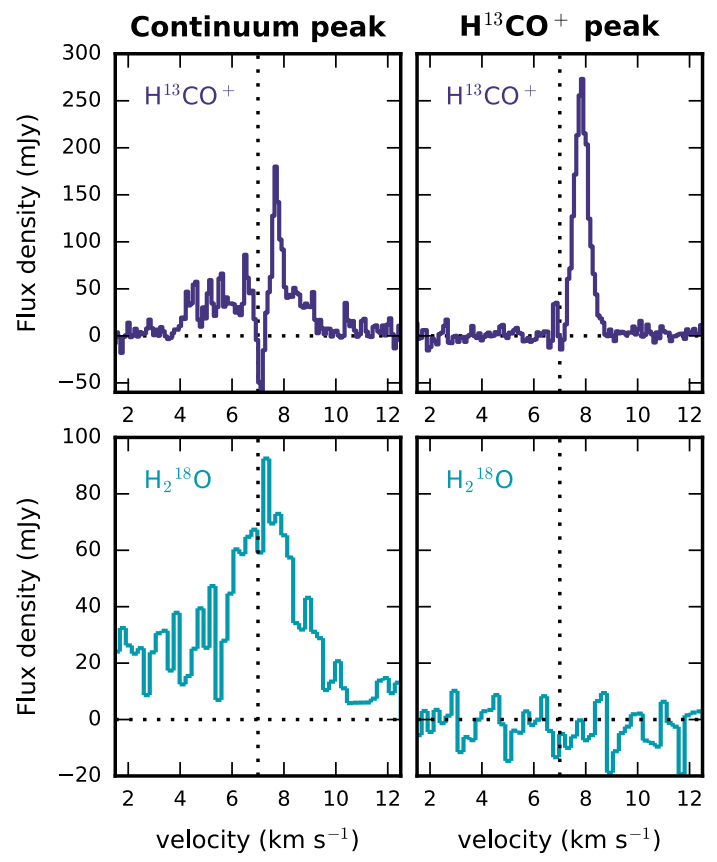

Fig. 4. Spectra extracted in a beam toward the continuum peak position (left panels) and the $\mathrm{H}^{13} \mathrm{CO}^{+}$peak position $\left(\sim 2^{\prime \prime} \mathrm{NE}\right.$ of the continuum peak, right panels). The top panels show the $\mathrm{H}^{13} \mathrm{CO}^{+} J$ $=3-2$ transition and the bottom panels the $\mathrm{H}_{2}^{18} \mathrm{O} 3_{1,3}-2_{2,0}$ transition. The two spectra for each species are shown on the same vertical scale. The systemic velocity is indicated by the vertical dotted line at $v_{\mathrm{lsr}} \approx 7 \mathrm{~km} \mathrm{~s}^{-1}$.

\section{Results}

The $\mathrm{H}^{13} \mathrm{CO}^{+}$channel maps presented in Fig. 1 show detection of $\mathrm{H}^{13} \mathrm{CO}^{+}$at velocities between 6.2 and $8.4 \mathrm{~km} \mathrm{~s}^{-1}$ $\left(v_{\mathrm{lsr}}=7.0 \mathrm{~km} \mathrm{~s}^{-1}\right.$; Persson et al. 2012), although the emission is brightest for the redshifted velocities. In none of the channels does the $\mathrm{H}^{13} \mathrm{CO}^{+}$emission peak at the source position. Instead, emission peaks are observed at offsets (RA, Dec) of approximately $\left(2^{\prime \prime}, 1.5^{\prime \prime}\right)$ and $\left(-3^{\prime \prime},-3^{\prime \prime}\right)$ in the redshifted channels, and at $\left(-1^{\prime \prime},-6^{\prime \prime}\right)$ in the blueshifted channels. $\mathrm{H}_{2}^{18} \mathrm{O}$, on the other hand, shows compact emission on source (located by Persson et al. 2012, $\sim 0$.' 1 southwest of the continuum peak) and extends along 
the southern outflow axis. Overlaying the integrated intensity maps for $\mathrm{H}^{13} \mathrm{CO}^{+}$and $\mathrm{H}_{2}^{18} \mathrm{O}$ (see Fig. 2) shows that the $\mathrm{H}^{13} \mathrm{CO}^{+}$ emission surrounds the water emission and peaks $\sim 2^{\prime \prime}$ northeast of the continuum peak.

The asymmetry in the spatial distribution of the $\mathrm{H}^{13} \mathrm{CO}^{+}$ emission is partly due to the sampling of the $(u, v)$ plane; simulating emission from a spherical envelope model with a ring-like $\mathrm{H}^{13} \mathrm{CO}^{+}$abundance distribution using the same $(u, v)$ coverage as the observations results in emission peaks in the northeast and southwest rather than a spherically symmetric emission pattern (see Fig. 3). The absence of a strong southwestern peak in the data may be attributed to the presence of water in the southern outflow. Alternatively, it could be an effect of the 3D structure of the source, which causes the $\mathrm{H}^{13} \mathrm{CO}^{+}$emission from that part to be blocked. Finally, it could perhaps be the result of asymmetries in the initial filamentary structure at large scales ( $\gg 10000 \mathrm{AU})$.

Spectra for $\mathrm{H}^{13} \mathrm{CO}^{+}$and $\mathrm{H}_{2}^{18} \mathrm{O}$ extracted toward the continuum and $\mathrm{H}^{13} \mathrm{CO}^{+}$peak positions are presented in Fig. 4. Although there is still $\mathrm{H}^{13} \mathrm{CO}^{+}$emission present on source, it is reduced by $\sim 40 \%$ compared to the region in which water emission is absent. The narrow width $\left(F W H M \approx 0.8 \mathrm{~km} \mathrm{~s}^{-1}\right)$ of the $\mathrm{H}^{13} \mathrm{CO}^{+}$line at its peak position indicates that the emission does not originate in the outflow. In comparison, the $\mathrm{H}_{2}^{18} \mathrm{O}$ line width is $\sim 3.5 \mathrm{~km} \mathrm{~s}^{-1}$. In addition, it has been shown in Persson et al. (2012) that the outflow component of the $\mathrm{H}_{2}^{18} \mathrm{O}$ emission can be distinguished spatially from the compact component. The emission around $v_{l s r}=3 \mathrm{~km} \mathrm{~s}^{-1}$ in the $\mathrm{H}_{2}^{18} \mathrm{O}$ spectrum is due to dimethyl ether $\left(\mathrm{CH}_{3} \mathrm{OCH}_{3}\right.$; Persson et al. 2012).

The absorption feature for $\mathrm{H}^{13} \mathrm{CO}^{+}$around the systemic velocity is partly because emission from the large-scale envelope is resolved out, as can be seen from the channel maps in Fig. 1 where most of the emission is absent around the systemic velocity. In addition, a comparison with JCMT single dish observations from Jørgensen et al. (2004b) shows that only $\sim 7 \%$ of the peak flux is recovered in the NOEMA observations, demonstrating that the bulk of the $\mathrm{H}^{13} \mathrm{CO}^{+}$is located on larger scales. Imaging the data before continuum subtraction shows that some absorption also occurs against the continuum in the central channels, which is indicative of cold gas along the line of sight at scales larger than the scale of interest.

\section{Analysis and discussion}

\subsection{Parametrized abundance profile for $\mathrm{H}^{13} \mathrm{CO}^{+}$}

To establish the origin of the dip in the $\mathrm{H}^{13} \mathrm{CO}^{+}$emission at the central position, $1 \mathrm{D}$ spherically symmetric physical-chemical modeling was performed using the radiative transfer code Ratran (Hogerheijde \& van der Tak 2000). For the physical structure we adopted the temperature and density profiles for IRAS2A from Kristensen et al. (2012), derived using the 1D spherically symmetric dust radiative transfer code DUSTY (Ivezić \& Elitzur 1997). In this procedure the free model parameters were fitted to the spectral energy distribution (SED) and the spatial extent of the sub-mm continuum emission. Based on the chemical consideration that $\mathrm{H}^{13} \mathrm{CO}^{+}$is particularly abundant when its main destructor $\mathrm{H}_{2} \mathrm{O}$ is frozen out, the simplest $\mathrm{H}^{13} \mathrm{CO}^{+}$distribution is an abundance profile with a low abundance inside the $100 \mathrm{~K}$ radius (i.e., the water snowline) and a higher abundance at larger radii. Both the inner and outer abundances are varied to get the best match to the observed integrated intensity along

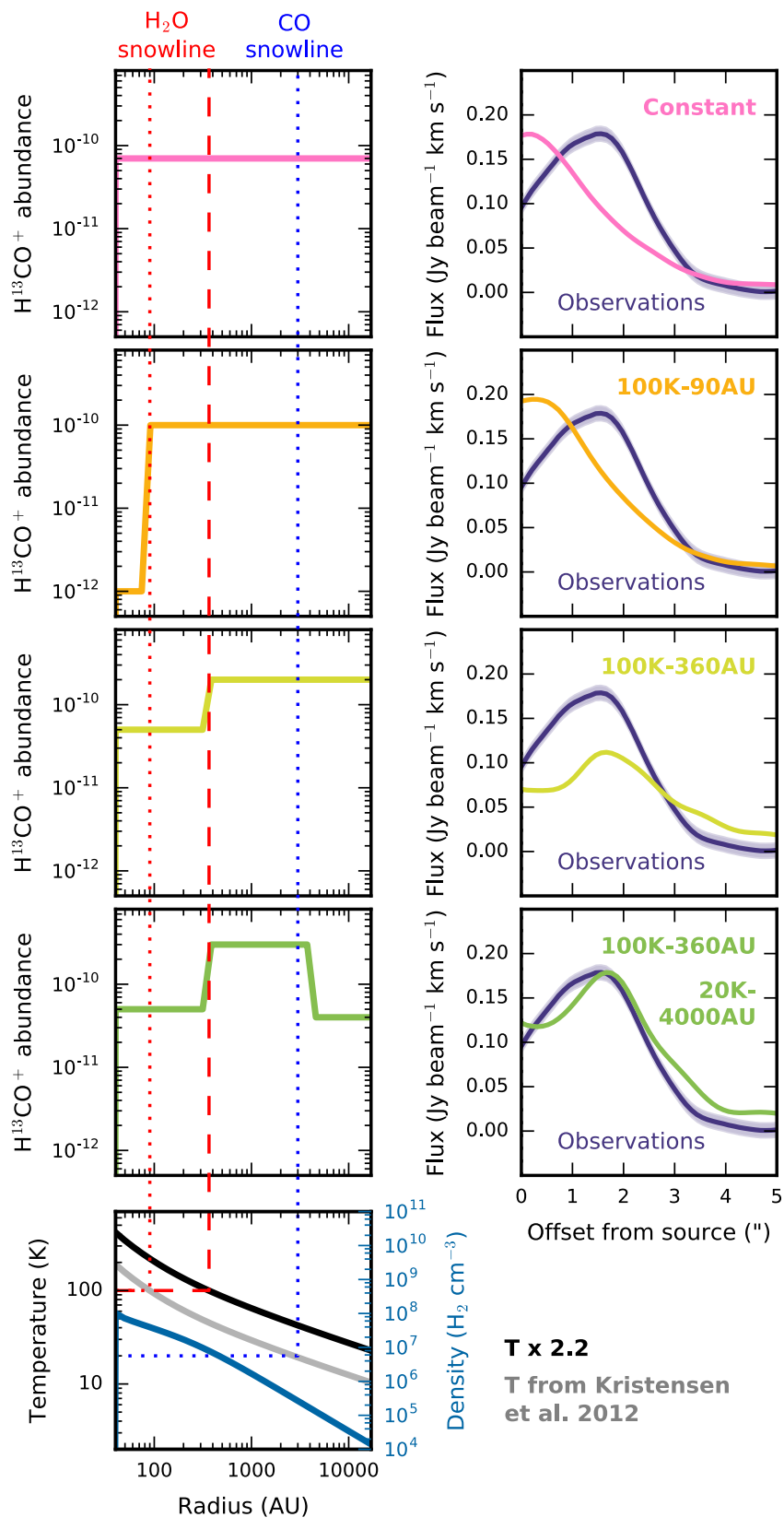

Fig. 5. Various $\mathrm{H}^{13} \mathrm{CO}^{+}$abundance profiles (left panels) and the resulting simulated integrated emission along the northeastern part of the radial cut shown in Fig. 2 (right panels). The observed emission is shown in purple. The location of the abundance jumps (in Kelvin and $\mathrm{AU}$ ) is indicated in the top right corners. Bottom panel: temperature (gray) and density (blue) profiles for the IRAS2A envelope from Kristensen et al. (2012) used in the top two models. The location of the $\mathrm{H}_{2} \mathrm{O}$ and $\mathrm{CO}$ snowlines (at $100 \mathrm{~K}$ and $20 \mathrm{~K}$, resp.) are indicated by the dotted red and blue lines, respectively. The temperature profile increased by a factor of 2.2, used in the bottom two models, is shown in black. The resulting $\mathrm{H}_{2} \mathrm{O}$ snowline is indicated by the dashed red line while the $\mathrm{CO}$ snowline now falls outside the adopted radial range. For IRAS2A, 1" corresponds to $\sim 250 \mathrm{AU}$.

the radial cut shown in Fig 2. The absence of the southwest $\mathrm{H}^{13} \mathrm{CO}^{+}$peak (discussed in Sect. 3) cannot be modeled with an axisymmetric 1D model. We therefore restrict the comparison to the northeastern part of the radial cut. For a first analysis, we do not adopt a velocity structure. The difference between the blue- and redshifted channels then cannot be reproduced exactly. Since there is almost no emission along the radial cut in the 

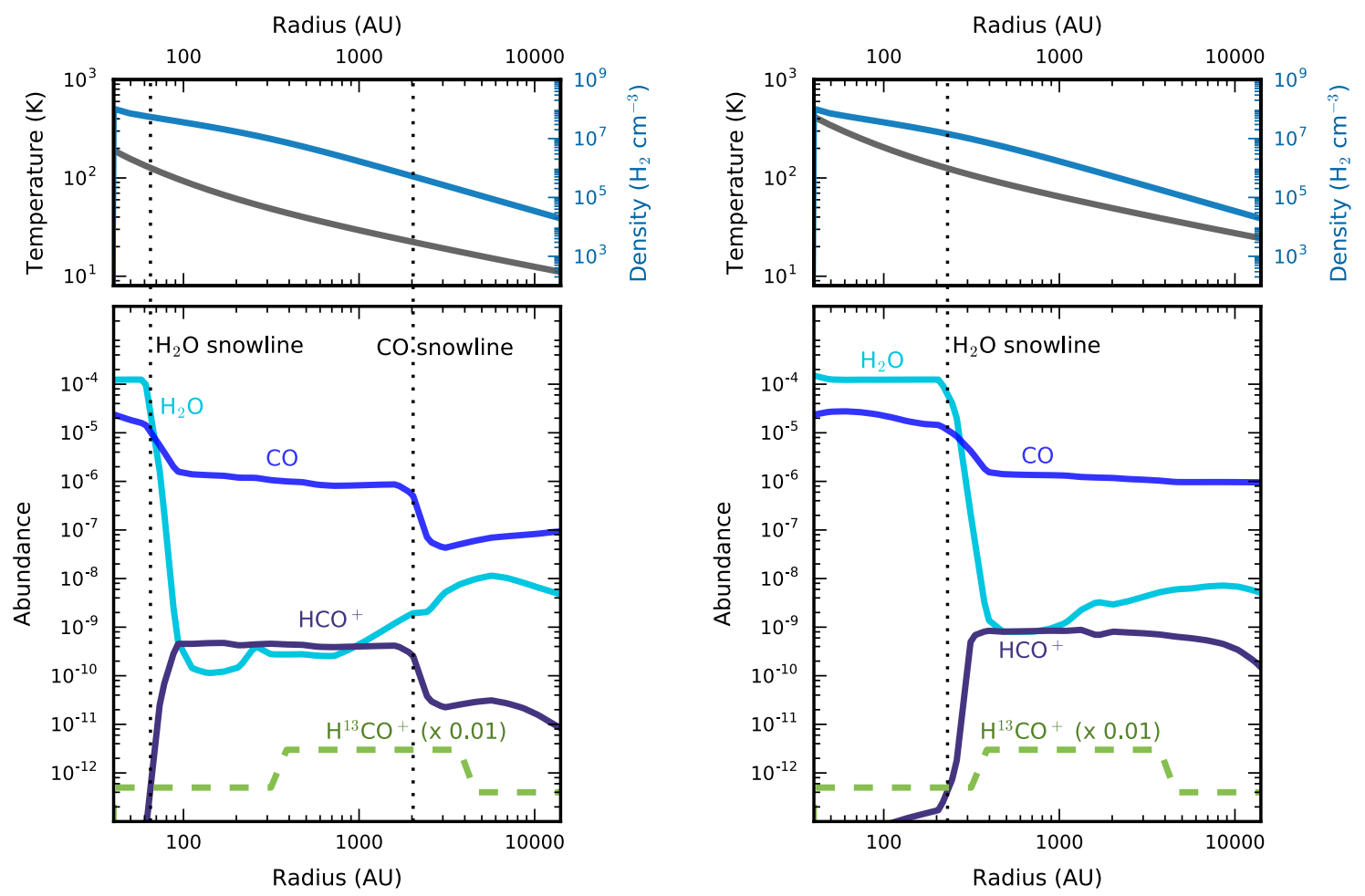

Fig. 6. Left panels: temperature (gray) and density (blue) profiles for the IRAS2A envelope from Kristensen et al. (2012) (top), and the corresponding $\mathrm{H}_{2} \mathrm{O}$ (light blue), $\mathrm{CO}$ (blue), and $\mathrm{HCO}^{+}$(purple) abundances predicted by the GRAINOBLE model (bottom). The dashed green line shows the empirically inferred abundance profile for $\mathrm{H}^{13} \mathrm{CO}^{+}$(scaled down by a factor 100). Right panels: as left panels, but with the temperature increased by a factor 2.2. The vertical dotted lines indicate the $\mathrm{H}_{2} \mathrm{O}$ and $\mathrm{CO}$ snowlines.

blueshifted channels, only the redshifted channels are used to construct the zeroth moment maps. Including all channels does not significantly change the observed integrated radial profile, but only affects the $\mathrm{H}^{13} \mathrm{CO}^{+}$abundance required to reach the observed intensity (by a factor of $\sim 3$ ). However, deriving the precise $\mathrm{H}^{13} \mathrm{CO}^{+}$abundance is not the goal of this work. Finally, simulated images representing the observed $(u, v)$ coverage are produced from the Ratran images in GILDAS by creating a $(u, v)$ table with the function uv_fmodel.

Various $\mathrm{H}^{13} \mathrm{CO}^{+}$abundance profiles with their corresponding integrated emission profiles are presented in Fig. 5. For the adopted physical structure, the temperature drops below $100 \mathrm{~K}$ around $90 \mathrm{AU}$. An $\mathrm{H}^{13} \mathrm{CO}^{+}$abundance of $1 \times 10^{-10}$ at radii $>90 \mathrm{AU}$ can roughly reproduce the observed integrated intensity (Fig. 5, second row). However, independent of the inner abundance, the emission always peaks on source, as in the case of a constant $\mathrm{H}^{13} \mathrm{CO}^{+}$abundance at all radii (Fig. 5, top row). Although a large percentage $(\sim 90 \%)$ of the emission is already being resolved out with the $(u, v)$ coverage of the observations, this thus does not result in a dip in the $\mathrm{H}^{13} \mathrm{CO}^{+}$emission toward the source position.

To reproduce the location of the emission peak, the abundance jump has to be shifted outward to a radius of $\sim 360 \mathrm{AU}$. To move the $100 \mathrm{~K}$ radius, representing the water snowline, this far out, the temperature profile has to be multiplied by a factor of 2.2 (Fig. 5, third row). However, the observed integrated intensity cannot be reached with this abundance profile, as the $\mathrm{H}^{13} \mathrm{CO}^{+}$emission becomes optically thick for outer abundances higher than $3 \times 10^{-10}$. Varying the abundance does not affect the position of the emission peak.

The best match to the observed $\mathrm{H}^{13} \mathrm{CO}^{+}$radial profile can be reached by introducing an additional drop in the abundance around $\sim 4000 \mathrm{AU}$ (Fig. 5, fourth row). An increase in abundance of at least a factor of six is required around $360 \mathrm{AU}$ to get the emission peak at the observed location, but the exact radius of the outer drop does not influence the peak position. Such a decrease in the $\mathrm{H}^{13} \mathrm{CO}^{+}$abundance can be expected around the $\mathrm{CO}$ snowline because formation of $\mathrm{HCO}^{+}$is limited in this region because of a reduction in the parent gaseous $\mathrm{CO}$ molecules. A radius of $4000 \mathrm{AU}$ corresponds to $17 \mathrm{~K}$ for the Kristensen et al. (2012) temperature profile, consistent with the $\mathrm{CO}$ freeze-out temperature for a pure $\mathrm{CO}$ ice binding energy. In contrast, for the increased temperature profile the temperature is $38 \mathrm{~K}$ at this radius; temperatures below $20 \mathrm{~K}$ are not reached within the $20000 \mathrm{AU}$ radial extent of the model. An increased temperature is thus required to set the inner radius of the $\mathrm{H}^{13} \mathrm{CO}^{+}$abundance at the water snowline, while the original temperature places the outer radius around the $\mathrm{CO}$ snowline. This discrepancy is further discussed in Sects. 4.2 and 4.3. The main result of these models is that the observed position of the $\mathrm{H}^{13} \mathrm{CO}^{+}$emission peak can only be reproduced by a reduction in the abundance of at least a factor 6 in the inner $\sim 360 \mathrm{AU}$.

\subsection{Comparison with a full chemical model}

To inspect the legitimacy of the simple abundance profile, we compared it to the outcome of the astrochemical code GRAINOBLE (Taquet et al. 2012a,b, 2013, 2014). In short, GRAINOBLE uses the rate equations approach (Hasegawa et al. 1992) considering three types of reactions (a so-called three-phase model based on Hasegawa \& Herbst 1993): reactions for gas-phase species, reactions for species on the ice surface, and reactions for bulk ice species. The gas-phase chemical network is taken from the KIDA database (Wakelam et al. 2015) supplemented by ion-neutral reactions described in Taquet et al. (2016). A cosmic 
ray (CR) ionization rate of $5 \times 10^{-17} \mathrm{~s}^{-1}$ is used. In addition to thermal desorption, UV photodesorption and chemical desorption upon formation of an exothermic surface reaction have been included as desorption processes. The surface chemical network follows the results of recent laboratory experiments to form the main ice species (see Taquet et al. 2013, for references). For $\mathrm{CO}$ and $\mathrm{H}_{2} \mathrm{O}$ binding energies have been adopted for an amorphous water ice substrate: $1150 \mathrm{~K}$ and $5775 \mathrm{~K}$, respectively (Collings et al. 2004; Fraser et al. 2001). The formation of interstellar ices is first followed during the formation of a dense starless core with the method described in Taquet et al. (2014). This ice chemical composition is then used as initial conditions for the gas-grain chemical evolution around IRAS2A, adopting the static 1D physical profile derived by Kristensen et al. (2012). Surface reactions have a negligible impact on the $\mathrm{HCO}^{+}$abundance during the latter protostellar phase. They have a significant importance during the former prestellar phase since they govern the transformation of $\mathrm{CO}$ ice into other species, thereby setting the initial ice abundance for the protostellar phase. The initial gas-phase abundances and ice abundances at the start of the protostellar phase are listed in Appendix A.

The left panel of Fig. 6 presents the $\mathrm{H}_{2} \mathrm{O}, \mathrm{CO}$ and $\mathrm{HCO}^{+}$ abundances for the IRAS2A envelope structure after $10^{4} \mathrm{yr}$ of chemical evolution. The abundance profiles do not change significantly for evolution up to $\sim 10^{5} \mathrm{yr}$, that is, the duration of the protostellar phase. Going from the outer envelope inward, the temperature rises above $20 \mathrm{~K}$ at a radius of $\sim 2700 \mathrm{AU}$, causing $\mathrm{CO}$ to desorb from the ice surface into the gas phase. In the three-phase model, a significant part of the icy $\mathrm{CO}$ remains trapped in the ice matrix at temperatures higher than the $\mathrm{CO}$ desorption temperature. These $\mathrm{CO}$ molecules evaporate together with water when the temperature exceeds $\sim 100 \mathrm{~K}$, resulting in a double abundance jump profile for $\mathrm{CO}$. The water snowline, that is, the radius where $50 \%$ of the water is in the gas phase and $50 \%$ is frozen out, is located at a distance of $\sim 65 \mathrm{AU}$ from the protostar (corresponding to $127 \mathrm{~K}$ ). As expected, the $\mathrm{HCO}^{+}$abundance increases sharply at radii beyond the water snowline, where its main destructor is frozen out, and decreases again outside the $\mathrm{CO}$ snowline, where the availability of its parent molecule is reduced. Increasing the temperature by a factor of 2.2 , as was required to match the observed $\mathrm{H}^{13} \mathrm{CO}^{+}$emission with a drop in the abundance at the $100 \mathrm{~K}$ radius, results in a similar chemical profile, only with the snowlines shifted to larger radii (Fig. 6, right panel). The water snowline is now located at $225 \mathrm{AU}(127 \mathrm{~K})$, while the $\mathrm{CO}$ snowline falls outside the modeled radial range. The water snowline location matches the observed radial extent of the $\mathrm{H}_{2}^{18} \mathrm{O}$ emission $\left(\sim 1^{\prime \prime} \approx 250 \mathrm{AU}\right)$.

For comparison, the best $\mathrm{H}^{13} \mathrm{CO}^{+}$abundance profile derived from the data is added to Fig. 6 (dashed green line). This inferred $\mathrm{H}^{13} \mathrm{CO}^{+}$abundance is higher than expected based on the GRAINOBLE results: similar to the predicted $\mathrm{HCO}^{+}$abundance instead of a factor $\sim 70$ lower. This can most likely be explained by the relatively low $\mathrm{CO}$ abundance between the $\mathrm{CO}$ and $\mathrm{H}_{2} \mathrm{O}$ snowlines in the chemical model, which directly results in a low $\mathrm{HCO}^{+}$abundance. The $\mathrm{CO}$ abundance is low because a large percentage of the $\mathrm{CO}$ ice is converted into methanol during the prestellar phase (Table A.2). In addition, in the three-phase model, not all $\mathrm{CO}$ evaporates inside its snowline, but remains trapped in water ice (Fig. 6). However, it is the overall trend that the $\mathrm{HCO}^{+}$abundance sharply rises at the $\mathrm{H}_{2} \mathrm{O}$ snowline and drops at the $\mathrm{CO}$ snowline that is important.
The radius where $\mathrm{H}^{13} \mathrm{CO}^{+}$has to increase (360 AU) matches the inner radius of the $\mathrm{HCO}^{+}$distribution in the chemical network with the increased temperature profile (right panel). The water snowline, however, is located $\sim 135 \mathrm{AU}$ closer to the star, at $225 \mathrm{AU}$. Similar behavior is seen in models for $\mathrm{N}_{2} \mathrm{H}^{+}$; the $50 \%$ reduction in $\mathrm{CO}$, or in this case $\mathrm{H}_{2} \mathrm{O}$, at the snowline is not yet sufficient to diminish the destruction of $\mathrm{N}_{2} \mathrm{H}^{+}$or $\mathrm{HCO}^{+}$, respectively, moving the emission ring outward (Aikawa et al. 2015; van 't Hoff et al. 2017). There are thus two effects at play here: first, the $\mathrm{HCO}^{+}$abundance is expected to peak outside rather than directly at the water snowline (360 vs. 225 AU). Second, this location for the water snowline is further away from the protostar than expected based on the Kristensen et al. (2012) temperature profile (225 vs. $65 \mathrm{AU}$ ).

The outer drop in $\mathrm{H}^{13} \mathrm{CO}^{+}$abundance matches less well with the predicted $\mathrm{HCO}^{+}$distributions. The best agreement is for the original temperature profile (for the adopted $\mathrm{CO}$ binding energy of $1150 \mathrm{~K})$, although $\mathrm{HCO}^{+}$decreases $\sim 2000 \mathrm{AU}(2.4 \mathrm{~K})$ further in than the radiative transfer modeling derived from the $\mathrm{H}^{13} \mathrm{CO}^{+}$data. Owing to the shallow gradient of the temperature profile in the outer envelope, the exact location of the $\mathrm{CO}$ snowline is very sensitive to small changes in the thermal structure. The mismatch could therefore be due to uncertainties in the derived temperature. Another important parameter for the snowline location is the assumed binding energy for $\mathrm{CO}$, which is related to the assumed ice structure, that is, whether $\mathrm{CO}$ ice is mixed with other molecules or pure. Adopting a lower binding energy, corresponding to a $\mathrm{CO}$ ice substrate rather than $\mathrm{a}_{2} \mathrm{O}$ ice substrate, would shift the snowline to larger radii. Anderl et al. (2016) investigated the CO binding energy using NOEMA observations of $\mathrm{C}^{18} \mathrm{O}$ and $\mathrm{N}_{2} \mathrm{H}^{+}$toward four young protostars and concluded that the extent of the $\mathrm{C}^{18} \mathrm{O}$ emission was best reproduced using a $\mathrm{CO}$ binding energy of $1200 \mathrm{~K}$, corresponding to a water ice substrate. In addition, these authors showed that the freeze-out temperature is $\sim 6 \mathrm{~K}$ lower assuming a binding energy of $855 \mathrm{~K}$ for pure CO ice instead of $1200 \mathrm{~K}$. Since the temperature difference between the $\mathrm{CO}$ snowline and the location of the $\mathrm{H}^{13} \mathrm{CO}^{+}$drop is only $2.4 \mathrm{~K}$, a high $\mathrm{CO}$ binding energy also seems best for IRAS2A. The small discrepancy between the CO snowline location predicted by the chemical model and the location inferred from the $\mathrm{H}^{13} \mathrm{CO}^{+}$observations may then be the result of asymmetries in IRAS2A, as hinted at by the asymmetric $\mathrm{C}^{18} \mathrm{O}$ emission shown by Anderl et al. (2016) for other protostars.

As noted before, the exact location of the drop in $\mathrm{H}^{13} \mathrm{CO}^{+}$ in the outer envelope does not influence the observed position of the emission peak. The main point is that the chemical model indeed predicts a steep rise in $\mathrm{HCO}^{+}$abundance just outside the water snowline, and a drop outside the $\mathrm{CO}$ snowline. This justifies the abundance profile used in the radiative transfer modeling, and corroborates that the depression in $\mathrm{H}^{13} \mathrm{CO}^{+}$in the center is due to the presence of gas-phase water.

\subsection{Tracing the CO snowline: signs of an accretion burst?}

Having the snowlines located further out than expected based on the adopted temperature profile derived from SED models, such as found here for the water snowline, could be a sign that the protostar has recently undergone a luminosity outburst. Such an outburst heats up the envelope, causing ices to sublimate off the grains. After the burst, the envelope cools rapidly (Johnstone et al. 2013), while it takes much longer for the molecules to freeze back onto the dust grains (Rodgers \& Charnley 2003). As a result, snowlines are shifted away from the star (Visser et al. 2015; Jørgensen et al. 2015; Cieza et al. 2016; 


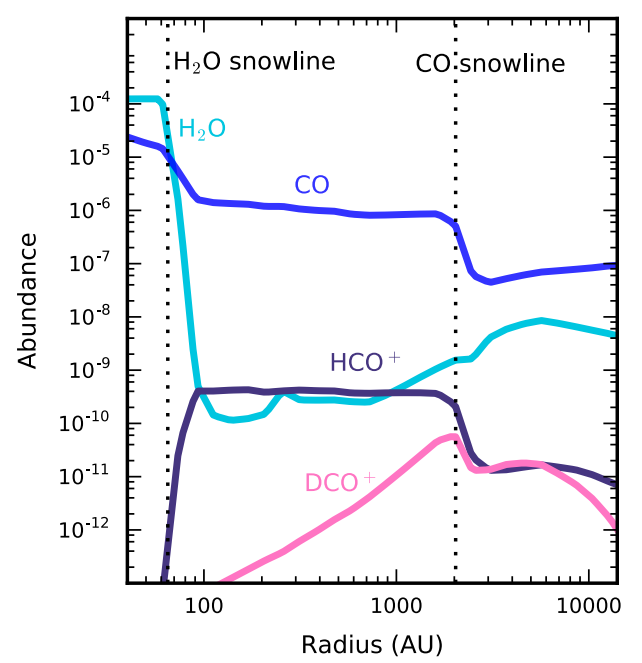

Fig. 7. $\mathrm{H}_{2} \mathrm{O}$ (light blue), $\mathrm{CO}$ (blue), $\mathrm{HCO}^{+}$(purple), and $\mathrm{DCO}^{+}$(pink) abundances predicted by the GRAINOBLE model for the IRAS2A temperature and density profile from Kristensen et al. (2012). The vertical dotted lines indicate the $\mathrm{H}_{2} \mathrm{O}$ and $\mathrm{CO}$ snowlines.

Frimann et al. 2017). An increased temperature structure is required to fit the $\mathrm{H}^{13} \mathrm{CO}^{+}$emission, suggesting that the water snowline is further out than expected. In case of an outburst, the CO snowline would also be shifted outward. Observational constraints for the $\mathrm{CO}$ snowline can thus help to establish whether IRAS2A has indeed recently undergone an accretion burst.

The deuterated form of $\mathrm{HCO}^{+}, \mathrm{DCO}^{+}$, can be used to trace the freeze-out of CO (e.g., Mathews et al. 2013). $\mathrm{DCO}^{+}$forms via reaction of $\mathrm{H}_{2} \mathrm{D}^{+}$and $\mathrm{CO}$. Formation of $\mathrm{H}_{2} \mathrm{D}^{+}$is enhanced at low temperatures $(<30 \mathrm{~K})$, but below $\sim 20 \mathrm{~K}$ CO starts to freeze-out. This balance results in $\mathrm{DCO}^{+}$peaking around the $\mathrm{CO}$ snowline (Murillo et al. 2015). Contamination of the emission by $\mathrm{DCO}^{+}$formed in warmer layers from $\mathrm{CH}_{2} \mathrm{D}^{+}$is not likely to be important in protostellar envelopes, unlike in protoplanetary disks (Favre et al. 2015). The $\mathrm{DCO}^{+}$abundance profile for the IRAS2A envelope structure is predicted with GRAINOBLE by extending the chemical network with a small network that includes reactions between $\mathrm{CO}$ and $\mathrm{H}_{2} \mathrm{D}^{+}, \mathrm{HD}_{2}^{+}$and $\mathrm{D}_{3}^{+}$. The $\mathrm{DCO}^{+}$abundance indeed peaks around the $\mathrm{CO}$ snowline (see Fig. 7), which is located at $\sim 2000$ AU for the fiducial temperature structure. As such, $\mathrm{DCO}^{+}$emission can be used to estimate the $\mathrm{CO}$ snowline location and constrain the temperature profile in the envelope.

The observed integrated intensity map for $\mathrm{DCO}^{+}$toward IRAS2A is presented in Fig. 8. Emission is observed south of the continuum peak and peaks $\sim 10^{\prime \prime}$ off source. Such an asymmetric emission pattern is observed for more protostars (e.g., Murillo et al. 2015). A CO snowline around 2000 AU, as predicted by the chemical model for the Kristensen et al. (2012) temperature profile, is in agreement with the $\mathrm{DCO}^{+}$emission. Thus, although the $\mathrm{H}_{2} \mathrm{O}$ snowline is located at larger radii than predicted based on the IRAS2A temperature profile ( $225 \mathrm{vs} .65 \mathrm{AU}$ ), the location of the $\mathrm{CO}$ snowline is as expected. Since the chemical reset after an outburst is faster at high densities, it is unlikely that the $\mathrm{CO}$ snowline has already shifted back while the $\mathrm{H}_{2} \mathrm{O}$ snowline has not. In addition, Jørgensen et al. (2015) presented $\mathrm{C}^{18} \mathrm{O}$ images obtained with the Submillimeter Array (SMA) for which the spatial extent matches well with the current luminosity. It is thus unlikely that IRAS2A has recently undergone an accretion burst. Why the temperature profile matches the snowline location for $\mathrm{CO}$ but not for $\mathrm{H}_{2} \mathrm{O}$ is further discussed in Sect. 4.5.

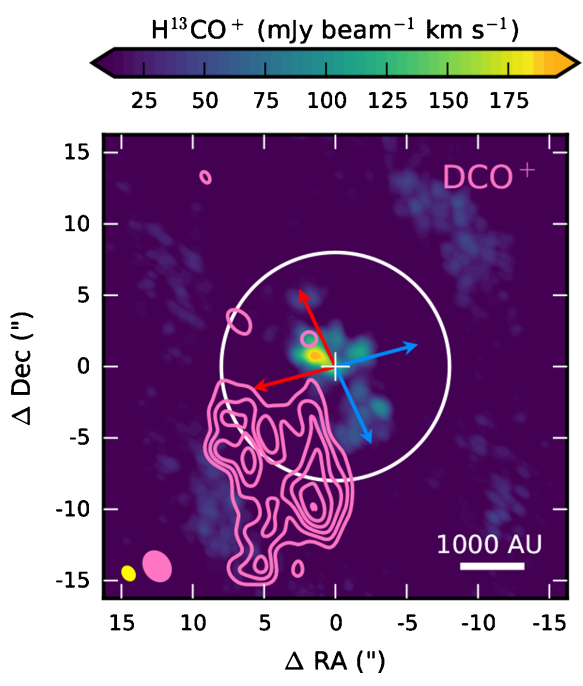

Fig. 8. Integrated intensity map for the $\mathrm{H}^{13} \mathrm{CO}^{+} J=3-2$ transition (color scale) toward IRAS2A. The $\mathrm{DCO}^{+} J=2-1$ transition is overlaid in pink contours. Contours are shown in steps of $1 \sigma$ starting from a $3 \sigma$ level $\left(15 \mathrm{mJy} \mathrm{beam}^{-1} \mathrm{~km} \mathrm{~s}^{-1}\right)$. The beams are depicted in the bottom left corner. The position of the continuum peak is indicated by a white cross and the outflow axes by red and blue arrows. The length of the arrows is the same as in Fig. 2 to compare the scales. The solid white contour represents the $\mathrm{CO}$ snowline radius predicted by the chemical model for the Kristensen et al. (2012) temperature profile ( 2000 AU).

\subsection{Attenuation of the cosmic ray ionization rate}

The chemistry leading to the formation of $\mathrm{HCO}^{+}$is initiated by the ionization of $\mathrm{H}_{2}$ molecules by cosmic rays (CRs). A reduction in the $\mathrm{CR}$ ionization rate therefore results in a lower $\mathrm{HCO}^{+}$ abundance. Numerical simulations performed by Padovani et al. (2013) showed that the CR ionization rate can be reduced by an order of magnitude in the inner region of a collapsing cloud core owing to twisting of the magnetic field lines. However, the exact value of the $\mathrm{CR}$ ionization rate in the inner regions of protostellar envelopes is unknown, and more recent work by Padovani et al. $(2015,2016)$ has shown that jet shocks and protostellar surface shocks can drive particle acceleration, leading to increased CR ionization rates.

Nonetheless, to assess if the effect of CR attenuation is large enough to cause the drop in $\mathrm{H}^{13} \mathrm{CO}^{+}$emission that we observe, we performed an order of magnitude calculation. As such, we approximate the reactions producing $\mathrm{H}_{3}^{+}$upon $\mathrm{CR}$ ionization by

$\zeta_{\mathrm{CR}}+\mathrm{H}_{2} \rightarrow \mathrm{H}_{3}^{+}$,

where $\zeta_{\mathrm{CR}}$ is the $\mathrm{CR}$ ionization rate. In this formalism we ignore $\mathrm{H}_{2}^{+}$, as the ionization is the rate limiting step for $\mathrm{H}_{3}^{+}$. Formation of $\mathrm{HCO}^{+}$is dominated by the reaction between $\mathrm{H}_{3}^{+}$and $\mathrm{CO}$,

$\mathrm{H}_{3}^{+}+\mathrm{CO} \rightarrow \mathrm{HCO}^{+}$,

and to estimate the importance of CRs, only recombination is considered as destruction reaction for $\mathrm{HCO}^{+}$, that is,

$\mathrm{HCO}^{+}+\mathrm{e}^{-} \rightarrow \mathrm{CO}+\mathrm{H}$.

Assuming steady state and $n\left(\mathrm{e}^{-}\right) \approx n\left(\mathrm{HCO}^{+}\right)$, the following relation can then be derived between the $\mathrm{HCO}^{+}$density, $n\left(\mathrm{HCO}^{+}\right)$, and the $\mathrm{CR}$ ionization rate, $\zeta_{\mathrm{CR}}$ (see Appendix $\mathrm{B}$ for more details):

$n\left(\mathrm{HCO}^{+}\right)=\sqrt{\frac{\zeta_{\mathrm{CR}} n\left(\mathrm{H}_{2}\right)}{k}}$, 
where $n\left(\mathrm{H}_{2}\right)$ is the molecular density of $\mathrm{H}_{2}$ and $k$ is the reaction rate coefficient for the $\mathrm{HCO}^{+}$destruction reaction (Eq. (4)). From this it can be seen that an order of magnitude reduction in the $\mathrm{CR}$ ionization rate gives a factor of three reduction in $\mathrm{HCO}^{+}$ abundance. Based on our radiative transfer modeling, at least a factor of six is required to fit the observations. Attenuation of the $\mathrm{CR}$ ionization rate alone is thus not likely to be the cause of the observed reduced $\mathrm{H}^{13} \mathrm{CO}^{+}$emission toward the center of IRAS2A.

\subsection{Physical structure}

Finally, a more likely explanation for the discrepancy between the temperatures needed for the $\mathrm{CO}$ and $\mathrm{H}_{2} \mathrm{O}$ snowlines may be that the physical structure of IRAS2A is more complicated than assumed with the 1D model. For several young protostars, it has been shown that a spherical envelope model cannot reproduce all of the continuum emission on small scales (e.g., Hogerheijde et al. 1999; Looney et al. 2003). This is also the case for IRAS2A (Brown et al. 2000; Jørgensen et al. 2005) and Persson et al. (2016) have derived the presence a compact disklike structure with a $167_{-21}^{+63}$ AU radius. The density may therefore change significantly on these scales of disk-envelope transition, that is, the scale at which we observe the $\mathrm{H}_{2}^{18} \mathrm{O}-\mathrm{H}^{13} \mathrm{CO}^{+}$anticorrelation. Since the envelope around IRAS2A is relatively optically thick to its own infrared radiation on $\sim 100$ AU scales, the exact temperature distribution becomes very dependent on the assumption about the density structure. In addition, the presence of an outflow cavity can shift the $100 \mathrm{~K}$ radius outward in specific directions (Bjerkeli et al. 2016), as the temperature along the cavity wall is higher at certain radii than in the envelope midplane (Visser et al. 2012). However, these considerations do not affect the main conclusion that the observations show a clear anticorrelation between $\mathrm{H}_{2}^{18} \mathrm{O}$ and $\mathrm{H}^{13} \mathrm{CO}^{+}$emission.

\section{Summary and outlook}

We have presented subarcsecond resolution observations of the $\mathrm{H}^{13} \mathrm{CO}^{+} J=3-2$ transition at $260.255 \mathrm{GHz}$ toward the Class 0 protostar IRAS2A, and for the first time, compared these directly to observations of $\mathrm{H}_{2}^{18} \mathrm{O}$, which were presented by Persson et al. (2012). While the $\mathrm{H}_{2}^{18} \mathrm{O} 3_{1,3}-2_{2,0}$ emission is centered at the continuum peak within a $\sim 1^{\prime \prime}$ radius, the $\mathrm{H}^{13} \mathrm{CO}^{+}$ emission peaks $\sim 2^{\prime \prime}$ to the northeast.

Using a 1D envelope model for IRAS2A, this offset in $\mathrm{H}^{13} \mathrm{CO}^{+}$emission can be explained by an abundance decrease of at least a factor of six in the inner $\sim 360 \mathrm{AU}$, but not by a constant $\mathrm{H}^{13} \mathrm{CO}^{+}$abundance. An increase in $\mathrm{H}^{13} \mathrm{CO}^{+}$abundance just outside the water snowline is consistent with chemical model predictions; the water snowline is then located around $225 \mathrm{AU}$, whereas the $\mathrm{H}^{13} \mathrm{CO}^{+}$abundance peaks $\sim 135 \mathrm{AU}$ further out at $360 \mathrm{AU}$. This snowline radius is larger than expected if the 1D spherically symmetric temperature profile from Kristensen et al. (2012) is adopted. $\mathrm{DCO}^{+}$observations place the $\mathrm{CO}$ snowline at the expected location, making an outburst scenario unlikely.

The combined observations of $\mathrm{H}^{13} \mathrm{CO}^{+}$and $\mathrm{H}_{2}^{18} \mathrm{O}$, together with radiative transfer and chemical modeling, thus provide proof of concept for the $\mathrm{HCO}^{+}-\mathrm{H}_{2} \mathrm{O}$ anticorrelation. The next step would be to corroborate this for more protostellar systems. Establishing $\mathrm{H}^{13} \mathrm{CO}^{+}$as a good tracer of the water snowline may allow localization of the snowline in protoplanetary disks for which direct detection is very difficult.
Acknowledgements. We would like to thank the referee for useful comments. In addition, we are grateful to the IRAM staff, in particular Miguel Montargès, for his help with the observations and reduction of the data, and Nadia Murillo and Arthur Bosman for useful discussions. Astrochemistry in Leiden is supported by the European Union A-ERC grant 291141 CHEMPLAN, by the Netherlands Research School for Astronomy (NOVA) and by a Royal Netherlands Academy of Arts and Sciences (KNAW) professor prize. M.L.R.H acknowledges support from a Huygens fellowship from Leiden University. M.V.P. postdoctoral position is funded by the ERC consolidator grant 614264 . V.T. has received funding from the European Union's Horizon 2020 research and innovation programme under the Marie Sklodowska-Curie grant agreement no. 664931. The research of J.K.J. is supported by European Union ERC Consolidator Grant "S4F" (grant agreement No 646908) and Centre for Star and Planet Formation funded by the Danish National Research Foundation.

\section{References}

Aikawa, Y., Furuya, K., Nomura, H., \& Qi, C. 2015, ApJ, 807, 120 Ali-Dib, M. 2017, MNRAS, 467, 2845

Ali-Dib, M., Mousis, O., Petit, J.-M., \& Lunine, J. I. 2014, ApJ, 785, 125 Anderl, S., Maret, S., Cabrit, S., et al. 2016, A\&A, 591, A3

Bergin, E. A., \& van Dishoeck, E. F. 2012, Roy. Soc. London Philos. Trans. Ser A, 370,2778

Bergin, E. A., Melnick, G. J., \& Neufeld, D. A. 1998, ApJ, 499, 777

Bjerkeli, P., Jørgensen, J. K., Bergin, E. A., et al. 2016, A\&A, 595, A39

Booth, R. A., Clarke, C. J., Madhusudhan, N., \& Ilee, J. D. 2017, MNRAS, 469, 3994

Brown, D. W., Chandler, C. J., Carlstrom, J. E., et al. 2000, MNRAS, 319, 154

Burke, D. J., \& Brown, W. A. 2010, Phys. Chem. Chem. Phys., 12, 5947

Cieza, L. A., Casassus, S., Tobin, J., et al. 2016, Nature, 535, 258

Collings, M. P., Anderson, M. A., Chen, R., et al. 2004, MNRAS, 354, 1133

Eistrup, C., Walsh, C., \& van Dishoeck, E. F. 2016, A\&A, 595, A83

Enoch, M. L., Young, K. E., Glenn, J., et al. 2006, ApJ, 638, 293

Favre, C., Bergin, E. A., Cleeves, L. I., et al. 2015, ApJ, 802, L23

Fraser, H. J., Collings, M. P., McCoustra, M. R. S., \& Williams, D. A. 2001, MNRAS, 327, 1165

Frimann, S., Jørgensen, J. K., Dunham, M. M., et al. 2017, A\&A, 602, A120

Harsono, D., Bruderer, S., \& van Dishoeck, E. F. 2015, A\&A, 582, A41

Hasegawa, T. I., \& Herbst, E. 1993, MNRAS, 263, 589

Hasegawa, T. I., Herbst, E., \& Leung, C. M. 1992, ApJS, 82, 167

Hogerheijde, M. R., \& van der Tak, F. F. S. 2000, A\&A, 362, 697

Hogerheijde, M. R., van Dishoeck, E. F., Salverda, J. M., \& Blake, G. A. 1999 ApJ, 513, 350

Hogerheijde, M. R., Bergin, E. A., Brinch, C., et al. 2011, Science, 334, 338

Ivezić, Z., \& Elitzur, M. 1997, MNRAS, 287, 799

Johnstone, D., Hendricks, B., Herczeg, G. J., \& Bruderer, S. 2013, ApJ, 765, 133

Jørgensen, J. K. 2004, A\&A, 424, 589

Jørgensen, J. K., \& van Dishoeck, E. F. 2010, ApJ, 710, L72

Jørgensen, J. K., Hogerheijde, M. R., van Dishoeck, E. F., Blake, G. A., \& Schöier, F. L. 2004a, A\&A, 413, 993

Jørgensen, J. K., Schöier, F. L., \& van Dishoeck, E. F. 2004b, A\&A, 416, 603

Jørgensen, J. K., Bourke, T. L., Myers, P. C., et al. 2005, ApJ, 632, 973

Jørgensen, J. K., Bourke, T. L., Myers, P. C., et al. 2007, ApJ, 659, 479

Jørgensen, J. K., Visser, R., Sakai, N., et al. 2013, ApJ, 779, L22

Jørgensen, J. K., Visser, R., Williams, J. P., \& Bergin, E. A. 2015, A\&A, 579, A23

Kristensen, L. E., van Dishoeck, E. F., Bergin, E. A., et al. 2012, A\&A, 542, A8

Looney, L. W., Mundy, L. G., \& Welch, W. J. 2003, ApJ, 592, 255

Madhusudhan, N., Amin, M. A., \& Kennedy, G. M. 2014, ApJ, 794, L12

Maret, S., Belloche, A., Maury, A. J., et al. 2014, A\&A, 563, L1

Mathews, G. S., Klaassen, P. D., Juhász, A., et al. 2013, A\&A, 557, A132

Maury, A. J., Belloche, A., André, P., et al. 2014, A\&A, 563, L2

Melnick, G. J. 2009, in Submillimeter Astrophysics and Technology: a Symposium Honoring Thomas, eds. G. Phillips, D. C. Lis, J. E. Vaillancourt, et al. ASP Conf. Ser., 417, 59

Mordasini, C., van Boekel, R., Mollière, P., Henning, T., \& Benneke, B. 2016, ApJ, 832, 41

Murillo, N. M., Bruderer, S., van Dishoeck, E. F., et al. 2015, A\&A, 579, A114

Öberg, K. I., Murray-Clay, R., \& Bergin, E. A. 2011, ApJ, 743, L16

Öberg, K. I., Guzmán, V. V., Furuya, K., et al. 2015, Nature, 520, 198

Padovani, M., Hennebelle, P., \& Galli, D. 2013, A\&A, 560, A114

Padovani, M., Hennebelle, P., Marcowith, A., \& Ferrière, K. 2015, A\&A, 582, L13

Padovani, M., Marcowith, A., Hennebelle, P., \& Ferrière, K. 2016, A\&A, 590, A8

Persson, M. V., Jørgensen, J. K., \& van Dishoeck, E. F. 2012, A\&A, 541, A39 
Persson, M. V., Jørgensen, J. K., \& van Dishoeck, E. F. 2013, A\&A, 549,

Persson, M. V., Harsono, D., Tobin, J. J., et al. 2016, A\&A, 590, A33 Phillips, T. G., van Dishoeck, E. F., \& Keene, J. 1992, ApJ, 399, 533

Qi, C., Öberg, K. I., Wilner, D. J., et al. 2013, Science, 341, 630

Qi, C., Öberg, K. I., Andrews, S. M., et al. 2015, ApJ, 813, 128

Rodgers, S. D., \& Charnley, S. B. 2003, ApJ, 585, 355

Schoonenberg, D., \& Ormel, C. W. 2017, A\&A, 602, A2

Stevenson, D. J., \& Lunine, J. I. 1988, Icarus, 75, 146

Taquet, V., Ceccarelli, C., \& Kahane, C. 2012a, ApJ, 748, L3

Taquet, V., Ceccarelli, C., \& Kahane, C. 2012b, A\&A, 538, A42

Taquet, V., Peters, P. S., Kahane, C., et al. 2013, A\&A, 550, A127

Taquet, V., Charnley, S. B., \& Sipilä, O. 2014, ApJ, 791, 1

Taquet, V., López-Sepulcre, A., Ceccarelli, C., et al. 2015, ApJ, 804, 81

Taquet, V., Wirström, E. S., \& Charnley, S. B. 2016, ApJ, 821, 46

Tobin, J. J., Dunham, M. M., Looney, L. W., et al. 2015, ApJ, 798, 61

van Dishoeck, E. F., Herbst, E., \& Neufeld, D. A. 2013, Chem. Rev., 113, 9043

van 't Hoff, M. L. R., Walsh, C., Kama, M., Facchini, S., \& van Dishoeck, E. F. 2017, A\&A, 599, A101

Visser, R., Kristensen, L. E., Bruderer, S., et al. 2012, A\&A, 537, A55

Visser, R., Bergin, E. A., \& Jørgensen, J. K. 2015, A\&A, 577, A102

Wakelam, V., \& Herbst, E. 2008, ApJ, 680, 371

Wakelam, V., Loison, J.-C., Herbst, E., et al. 2015, ApJS, 217, 20

Walsh, C., Nomura, H., \& van Dishoeck, E. 2015, A\&A, 582, A88

Walsh, C., Loomis, R. A., Öberg, K. I., et al. 2016, ApJ, 823, L10

Zhang, K., Pontoppidan, K. M., Salyk, C., \& Blake, G. A. 2013, ApJ, 766,

\section{Appendix A: Initial abundances in the GRAINOBLE model}

Table A.1 lists the initial elemental abundances in the gas phase for the prestellar phase of the GRAINOBLE model, based on the low-metal model from Wakelam \& Herbst (2008). The ice composition at the end of this phase (after 1.7 Myr) is listed in Table A.2. These are the initial conditions for the chemical evolution around IRAS2A.

Table A.1. Initial gas-phase elemental abundances.

\begin{tabular}{lc}
\hline \hline Species & Abundance $^{a}$ \\
\hline $\mathrm{H}_{2}$ & 0.5 \\
$\mathrm{HD}$ & $1.5 \times 10^{-5}$ \\
$\mathrm{H}$ & $1.6 \times 10^{-4}$ \\
$\mathrm{He}$ & $9.0 \times 10^{-2}$ \\
$\mathrm{C}+$ & $7.3 \times 10^{-5}$ \\
$\mathrm{~N}$ & $2.1 \times 10^{-5}$ \\
$\mathrm{O}$ & $1.8 \times 10^{-4}$ \\
$\mathrm{Si}$ & $8.0 \times 10^{-9}$ \\
$\mathrm{~S}$ & $8.0 \times 10^{-8}$ \\
$\mathrm{Fe}$ & $3.0 \times 10^{-9}$ \\
$\mathrm{Na}$ & $2.0 \times 10^{-9}$ \\
$\mathrm{Mg}$ & $7.0 \times 10^{-9}$ \\
$\mathrm{Cl}$ & $1.0 \times 10^{-9}$ \\
\hline
\end{tabular}

Notes. ${ }^{(a)}$ Abundances with respect to the total number of hydrogen nuclei.

\section{Appendix B: Effect of an attenuated cosmic ray ionization rate}

A first order approximation of the effect of CR ionization rate on the $\mathrm{HCO}^{+}$abundance can be made by considering the main formation and destruction reactions of $\mathrm{HCO}^{+}$. Assuming steady state, that is, assuming that the $\mathrm{HCO}^{+}$abundance does not
Table A.2. Ice chemical composition at the end of the prestellar phase.

\begin{tabular}{lc}
\hline \hline Species & Abundance \\
\hline $\mathrm{H}_{2} \mathrm{O}$ & $1.1 \times 10^{-4}$ \\
$\mathrm{CO}$ & $1.3 \times 10^{-5}$ \\
$\mathrm{CO}_{2}$ & $1.7 \times 10^{-7}$ \\
$\mathrm{~N}_{2}$ & $9.8 \times 10^{-6}$ \\
$\mathrm{H}_{2} \mathrm{CO}$ & $6.7 \times 10^{-5}$ \\
$\mathrm{CH}_{3} \mathrm{OH}$ & $5.5 \times 10^{-5}$ \\
$\mathrm{NH}_{3}$ & $1.3 \times 10^{-6}$ \\
$\mathrm{CH}_{4}$ & $4.4 \times 10^{-6}$ \\
\hline
\end{tabular}

Notes. ${ }^{(a)}$ Abundances with respect to the total number of hydrogen nuclei.

change over time, we can write

$\frac{\mathrm{d} n\left(\mathrm{HCO}^{+}\right)}{\mathrm{d} t}=$ formation rate - destruction rate $=0$.

The main formation pathway of $\mathrm{HCO}^{+}$is through reaction between $\mathrm{CO}$ and $\mathrm{H}_{3}^{+}$,

$\mathrm{H}_{3}^{+}+\mathrm{CO} \rightarrow \mathrm{HCO}^{+}$,

and recombination with electrons is the main destruction route,

$\mathrm{HCO}^{+}+\mathrm{e}^{-} \rightarrow \mathrm{CO}+\mathrm{H}$.

The reaction of $\mathrm{HCO}^{+}$with $\mathrm{H}_{2} \mathrm{O}$ is ignored in this calculation to assess the importance of the $\mathrm{CR}$ ionization rate.

The rate of these reactions can be written as

rate $=k \times n(A) n(B)$,

where $k$ is the rate coefficient, and $n(A)$ and $n(B)$ are the molecular densities of the reacting species. Equation (B.1) then becomes

$k_{1} n\left(\mathrm{H}_{3}^{+}\right) n(\mathrm{CO})-k_{2} n\left(\mathrm{HCO}^{+}\right) n\left(\mathrm{e}^{-}\right)=0$.

The $\mathrm{H}_{3}^{+}$density, $n\left(\mathrm{H}_{3}^{+}\right)$, can be calculated by following the same procedure. The formation reactions are

$\mathrm{H}_{2}+\zeta_{\mathrm{CR}} \rightarrow \mathrm{H}_{2}^{+}+\mathrm{e}^{-}$, and

$\mathrm{H}_{2}^{+}+\mathrm{H}_{2} \rightarrow \mathrm{H}_{3}^{+}+\mathrm{H}$.

In order to solve Eq. (B.5) analytically, we summarize the formation of $\mathrm{H}_{3}^{+}$as follows:

$\zeta_{\mathrm{CR}}+\mathrm{H}_{2} \rightarrow \mathrm{H}_{3}^{+}$,

because the ionization reaction is the rate limiting step. In addition, only the reaction with $\mathrm{CO}$ (Eq. (B.3)) is considered as destruction mechanism. We can then write an equation similar to Eq. (B.5), that is,

$\zeta_{\mathrm{CR}} n\left(\mathrm{H}_{2}\right)-k_{1} n\left(\mathrm{H}_{3}^{+}\right) n(\mathrm{CO})=0$.

Substituting Eq. (B.9) into Eq. (B.5), and assuming $n\left(\mathrm{e}^{-}\right) \approx n\left(\mathrm{HCO}^{+}\right)$, we can solve Eq. (B.5) to get the following expression for the $\mathrm{HCO}^{+}$density:

$n\left(\mathrm{HCO}^{+}\right)=\sqrt{\frac{\zeta_{\mathrm{CR}} n\left(\mathrm{H}_{2}\right)}{k_{2}}}$. 